\title{
ESTRUTURA DA COMUNIDADE DE PEIXES NA ÁREA DE INFLUÊNCIA DIRETA DA PEQUENA CENTRAL HIDRELÉTRICA BRAÇO, RJ/SP
}

\author{
J. C. Miranda ${ }^{1}$, C.G.W. Ferreira ${ }^{2}$ e D.C. Andrade ${ }^{3}$ \\ ${ }^{1}$ Universidade Federal Fluminense \\ ${ }^{2}$ Instituto de Estudos do Mar Almirante Paulo Moreira \\ ${ }^{3}$ Instituto Alberto Luiz Coimbra de Pós-Graduação (COPPE/UFRJ) \\ E-mail: jeanmiranda@id.uff.br ${ }^{1}$, cgustavoferreira@gmail.com ${ }^{2}$, campbell.dan@hotmail.com ${ }^{3}$
}

Artigo submetido em março/2013 e aceito em novembro/2013

\section{RESUMO}

O presente trabalho objetivou caracterizar a estrutura da comunidade de peixes do rio do Braço na área de influência direta da $\mathrm{PCH}$ Braço, localizada entre os municípios de Bananal (SP) e Rio Claro (RJ), durante o primeiro ano de operação do empreendimento. Para tal, foram realizadas coletas trimestrais em seis pontos amostrais com redes de espera de $4 \mathrm{~m}$ de comprimento por $1 \mathrm{~m}$ de altura (entrenós adjacentes de $15 \mathrm{~mm}$ e 45 $\mathrm{mm}$ ) e peneiras. Foram capturados 272 indivíduos, pertencentes a sete espécies. Astyanax intermedius foi a espécie mais abundante ( $57 \%$ do total capturado), seguida por Neoplecostomus microps (17\% do total capturado) e Phalloceros sp. ( $12 \%$ do total capturado). Os maiores valores de riqueza e diversidade foram registrados em novembro de 2011 e fevereiro de 2012. A equitabilidade alcançou maior valor em agosto de 2011. Os resultados das análises de similaridade indicam a separação das coletas em dois grupos: maio e agosto de 2011 (período seco); novembro de 2011 e fevereiro de 2012 (período chuvoso).

PALAVRAS-CHAVE: Ictiofauna, Estrutura de comunidades, Pequenas Centrais Hidrelétricas, Mata Atlântica.

\section{FISH COMMUNITY STRUCTURE IN THE AREA UNDER DIRECT INFLUENCE OF SMALL HYDROELETRIC CENTRAL BRAÇO, RJ/SP}

\section{ABSTRACT}

The present study aimed characterize the fish community structure in the Braço River area of direct influence of the Small Hydroelectric Central Braço, located between the municipalities of Bananal (SP) and Rio Claro (RJ), during the first year of operation of the enterprise. For this purpose, it was sampled quarterly three sites with gillnets of 4 meter of length $x 1$ meter of height (adjacent internodes of $15 \mathrm{~mm}$ and $45 \mathrm{~mm}$ ) and sieves. Were captured 272 individuals belonging to seven species. Astyanax intermedius was the most abundant species (57\% of the total catch), followed by Neoplecostomus microps ( $17 \%$ of total catch) and Phalloceros sp. ( $12 \%$ of the total catch). The highest values of richness and diversity were registered in November 2011 and February 2012. The highest equitability reached in August 2011. Analysis of similarity indicated two groups: May and August of 2011 (rain season) and November 2011 and February of 2012 (dry season).

KEYWORDS: Ichthyofauna, community structure, small hydroelectric system, Atlantic Forest. 


\section{ESTRUTURA DA COMUNIDADE DE PEIXES NA ÁREA DE INFLUÊNCIA DIRETA DA PEQUENA CENTRAL HIDRELÉTRICA BRAÇO, RJ/SP}

\section{INTRODUÇÃO}

O represamento de rios é um fenômeno global (MOYLE e MOUNT, 2007). Há cerca de 45.000 grandes barragens no mundo (WWF, 2004) utilizadas para fins diversos: geração de eletricidade, abastecimento, irrigação, pesca, lazer e navegação (TUNDISI, 1999). Aproximadamente $60 \%$ dos grandes rios do planeta estão impactados por esse tipo de ação (PETESSE e PETRERE JÚNIOR, 2012).

A matriz energética brasileira é predominantemente hidrelétrica. 0 país tem a terceira maior capacidade hidrelétrica das Américas, atrás apenas dos Estados Unidos e Canadá, com 247 grandes represas destinadas a produção de energia elétrica (PETESSE e PETRERE JÚNIOR, 2012).

Em função do menor prazo de implementação e legislação mais acessível (KUSMA e FERREIRA, 2010), quando comparadas à grandes empreendimentos, as Pequenas Centrais Hidrelétricas ( $P C H s$ ) têm sido apontadas como alternativa para a geração de energia em rios de pequeno ou médio porte. Todavia, o desvio temporário do curso de rios e a retirada da vegetação para o estabelecimento de barragens podem desencadear processos com repercussões ecológicas, econômicas e sociais em diferentes escalas (SILVA NETO et al. 2011), além de modificar as condições ambientais interferindo nas comunidades biológicas aquáticas (MIRANDA, 2012).

A ictiofauna é a porção mais visível dessa comunidade e pode ser considerada como um bom bioindicador da qualidade geral do ambiente aquático (ARAÚJO, 1998; SHIBATTA et al., 2006). Segundo Carvalho e Silva (1999), os principais impactos negativos sobre as comunidades de peixes são as alterações na sua composição e estrutura. Espécies de peixes com hábitos migratórios (reofílicas), i.e. que apresentam orientação e movimento contra o escoamento (PAVLOV, 1989), por exemplo, podem sofrer impacto devido à formação de reservatórios para produção hidrelétrica, podendo ser extintas à montante da barragem por não realizarem a piracema.

Desta maneira, os estudos realizados antes, durante e após o represamento dos rios são importantes para o entendimento das possíveis mudanças na ictiofauna e o estabelecimento de propostas amenizadoras dos possíveis impactos decorrentes do empreendimento.

O presente trabalho tem como objetivo caracterizar a estrutura da comunidade de peixes do rio do Braço, na área de influência direta da $\mathrm{PCH}$ Braço durante o primeiro ano de operação do empreendimento.

\section{METODOLOGIA}

\section{Área de Estudo}

A PCH Braço está localizada no rio do Braço (Figura 1), bacia hidrográfica do Rio Paraíba do Sul. A área de influência da PCH Braço (22 47'S, 04414'W) situa-se, nos municípios de Rio Claro (RJ) e Bananal (SP), em contrafortes da Serra do Mar localizados em altitudes superiores a 1000 metros, mais precisamente na Serra da Carioca, inserida no domínio da Mata Atlântica, 
bioma ameaçado e considerado um dos 25 "hotspot" do mundo em biodiversidade (MYERS et al., 2000).

A região apresenta paisagem marcada por áreas montanhosas, com rios encaixados nas falhas e fraturas do relevo que correm de noroeste para nordeste. Devido às características do rio do Braço, em especial da área onde foi instalada a PCH Braço, espera-se que a construção da barragem não apresente prejuízo para as espécies reofílicas, pois as séries de cascatas existentes no rio já funcionam como barreira geográfica natural.

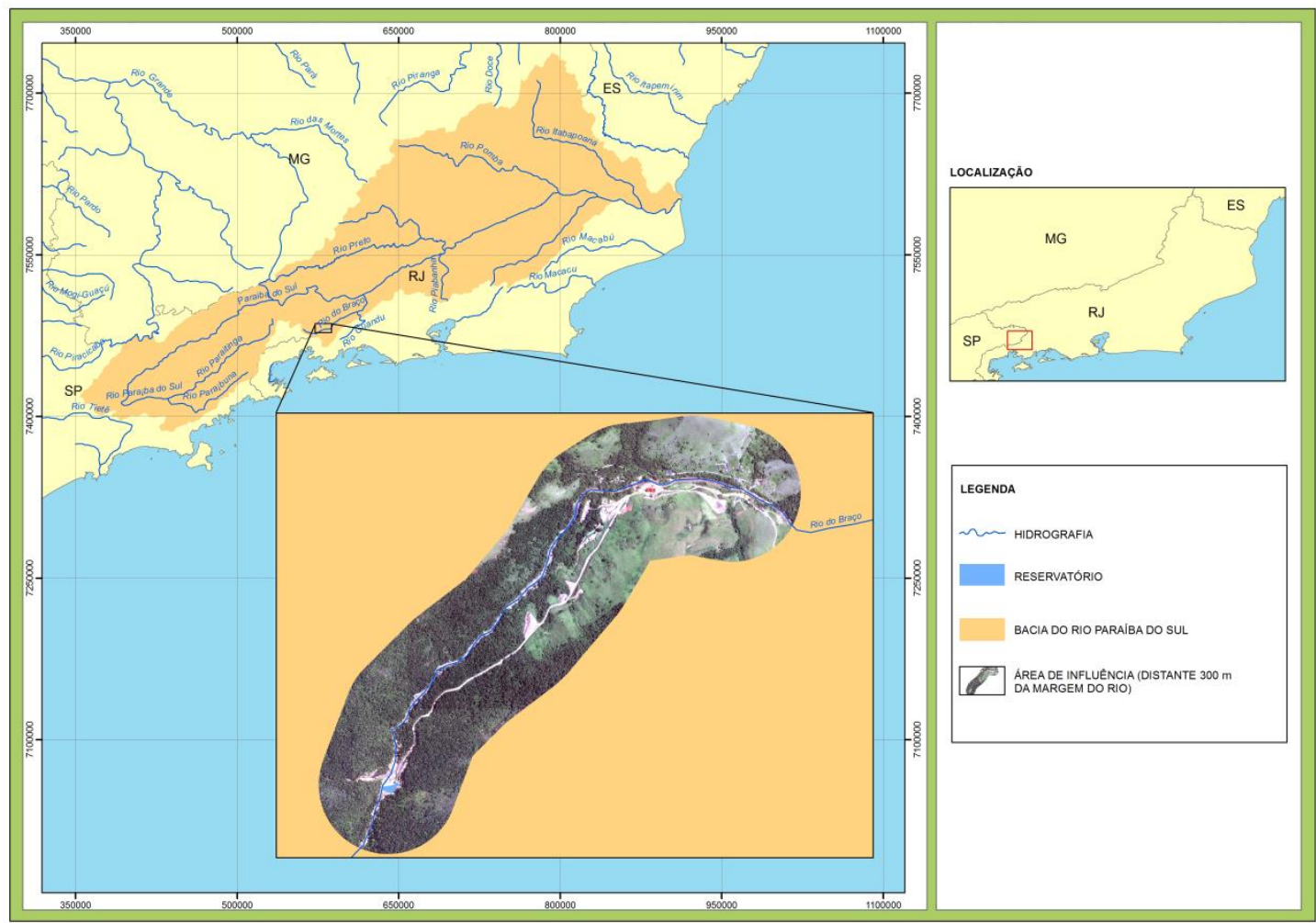

Figura 1 - Mapa de localização da área de estudo.

\section{Amostragem}

A obtenção dos dados sobre a composição e estrutura da ictiofauna se deu por meio de coletas trimestrais, entre maio de 2011 e fevereiro de 2012, em seis pontos amostrais (Tabela 1). Para amostragem o esforço foi padronizado, com utilização de redes de espera de $4 \mathrm{~m}$ de comprimento por $1 \mathrm{~m}$ de altura (entrenós adjacentes de $15 \mathrm{~mm}$ e $45 \mathrm{~mm}$ ). As redes ficaram expostas por um período de 48 horas, com vistorias a cada 12 horas. Foram realizadas coletas adicionais com utilização de peneiras e puçás. Os peixes coletados foram fixados em solução de formol a $10 \%$ e transportados ao laboratório para identificação ao menor nível taxonômico possível, com auxilio de bibliografia especializada (Pereira et al., 2000; Bizerril \& Primo 2001; Melo, 2001; Reis et al., 2006; Lucinda, 2008). Exemplares testemunhos foram depositados na Coleção Ictiológica do Museu Nacional - UFRJ (MNRJ39354, MNRJ39355, MNRJ39361, MNRJ39362, MNRJ39363, MNRJ39364, MNRJ39365). A licença para coleta foi concedida pelo Instituto Brasileiro de Meio Ambiente (Processo IBAMA no 02001.000336/2009-34, autorização no 080/2011 - GGFAP/IBAMA). 
Tabela 1: Coordenadas dos pontos amostrais na área de influência direta da PCH Braço.

\begin{tabular}{|c|c|c|}
\hline Pontos Amostrais & Coordenadas & Descrição \\
\hline \multirow{2}{*}{1} & S $22^{\circ} 47^{\prime} 39.2^{\prime \prime}$ & Área a montante do reservatório. \\
\hline & W $044^{\circ} 14^{\prime} 25.2^{\prime \prime}$ & (distante cerca de 340 m, em linha reta, do reservatório) \\
\hline \multirow{2}{*}{2} & S $22^{\circ} 47^{\prime} 29.4^{\prime \prime}$ & \multirow{2}{*}{ Área do reservatório da PCH Braço. } \\
\hline & W $044^{\circ} 14^{\prime} 21.3^{\prime \prime}$ & \\
\hline \multirow{2}{*}{3} & $S 22^{\circ} 47^{\prime} 27.2^{\prime \prime}$ & Área a jusante da barragem. \\
\hline & W $044^{\circ} 14^{\prime} 21.3^{\prime \prime}$ & (distante cerca de 60 m, em linha reta, do reservatório) \\
\hline \multirow{2}{*}{4} & $\mathrm{~S} 22^{\circ} 46^{\prime} 53.6^{\prime \prime}$ & Área a jusante da barragem. \\
\hline & W $044^{\circ} 13^{\prime} 56.2^{\prime \prime}$ & (distante cerca de $1.300 \mathrm{~m}$, em linha reta, do reservatório) \\
\hline \multirow{2}{*}{5} & $\mathrm{~S} 22^{\circ} 46^{\prime} 47.8^{\prime \prime}$ & Área a montante da casa de força. \\
\hline & W $044^{\circ} 13^{\prime} 45.6^{\prime \prime}$ & (distante cerca de $1.600 \mathrm{~m}$, em linha reta, do reservatório) \\
\hline \multirow{2}{*}{6} & S $22^{\circ} 46^{\prime} 47.2^{\prime \prime}$ & Área a jusante do canal de fuga. \\
\hline & W $044^{\circ} 13^{\prime} 42.2^{\prime \prime}$ & (distante cerca de $1.700 \mathrm{~m}$, em linha reta, do reservatório) \\
\hline
\end{tabular}

\section{Tratamento dos dados}

Os dados levantados foram analisados seguindo os princípios gerais de estudos de comunidades e agrupados por campanha de coleta $(\mathrm{C} 1=$ campanha $1-\mathrm{Maio} / 2011 ; \mathrm{C} 2=$ campanha 2 - Agosto/2011; C3 = campanha 3 - Novembro/2011; C4 = campanha 4 Fevereiro/2012). A estrutura das comunidades de peixes foi avaliada através dos seguintes atributos: (i) composição específica, (ii) riqueza, (iii) abundância relativa, (iv) diversidade (índice de Shannon-Wiener), (v) equitabilidade e (vi) similaridade (índices de Jaccard e Bray-Curts).

O cálculo da abundância relativa (definida como número de indivíduos de uma determinada espécie existente numa dada área num dado período de tempo, calculado em relação ao número total de indivíduos de todas as espécies existentes nessa área) de cada táxon identificado foi realizado aplicando a seguinte fórmula:

$$
A=N \times 100 / n
$$

onde: $\mathrm{N}$ = número de indivíduo do táxon identificado e $\mathrm{n}=$ número total de indivíduos.

Para interpretação da abundância relativa de cada táxon foi utilizada a seguinte classificação: Espécie rara: < 10\%; Espécie pouco abundante: 10 a 30\%; Espécie abundante: 30 a $50 \%$ e Dominante: $>50 \%$.

A diversidade específica e índices associados (i.e. riqueza e equitabilidade) foram verificados, para cada campanha, através do índice de Shannon-Wiener (MAGURRAN, 2004), representado pelas expressões:

$$
H=-\sum p i \log p i \quad \text { e } E=H /(\log S) \text {. }
$$


onde: $\mathrm{H}$ = diversidade; $\mathrm{pi}=$ proporção de indivíduos da espécie $\mathrm{i} ; \mathrm{E}=$ equitabilidade; $\log \mathrm{S}=$ diversidade máxima; $\mathrm{S}=$ número de espécies.

A similaridade entre as campanhas de coleta foi avaliada em seus aspectos qualitativos e quantitativos. Em termos qualitativos foi aplicado o índice de similaridade de Jaccard $(\mathrm{J})$ que utiliza apenas a presença e a ausência das espécies. É calculado pela fórmula:

$$
\mathrm{J}=\mathrm{c} / \mathrm{a}+\mathrm{b}-\mathrm{c}
$$

sendo: $\mathrm{J}=$ coeficiente; $\mathrm{a}=$ número de espécies presentes na campanha $\mathrm{a} ; \mathrm{b}=$ número de espécies presentes na campanha $b ; c=$ número de espécies comuns às campanhas $a$ e $b$.

Para análise em termos quantitativos, ou seja, utilizando os dados de abundância da ictiofauna, foi empregado o índice de Bray-Curtis (B) (BRAY e CURTIS, 1957), calculado por:

$$
B=\sum\left(X_{i j}-X_{i k}\right) / \sum\left(X_{i j}+X_{i k}\right)
$$

sendo: $X_{i j}=$ frequência de ocorrência da espécie $i$ na campanha $j ; X_{i k}=$ frequência de ocorrência da espécie $i$ na campanha $k$; 1-B= similaridade de Bray-Curtis.

\section{RESULTADOS E DISCUSSÃO}

Durante o monitoramento da ictiofauna na área de influência da PCH Braço no primeiro ano de sua operação, foram capturados 272 indivíduos, pertencentes a sete espécies: Astyanax intermedius Eigenmann, 1908; Characidium sp.; Hemipsilichthys gobio (Lütken, 1874); Hemipsilichthys papillatus Pereira, Oliveira \& Oyakawa, 2000; Neoplecostomus microps (Steindachner, 1877); Phalloceros sp. e Trichomycterus vermiculatus (Eigenmann, 1917).

O predomínio de Characiformes e Siluriformes (85,7\% da composição de espécies) na área de influência da $\mathrm{PCH}$ Braço segue o padrão esperado para sistemas fluviais sul-americanos (LOWE-MCCONNEL, 1987; CASTRO, 1999). Os valores registrados na área amostrada são semelhantes aos encontrados em outros estudos (e.g. BRAGA e ANDRADE, 2005; PEREZ-JUNIOR e GARAVELO, 2007),

Astyanax intermedius foi a espécie mais abundante (155 indivíduos; $57 \%$ do total capturado - Espécie dominante), seguida por Neoplecostomus microps (47 indivíduos; $17 \%$ do total capturado - Espécie pouco abundante) e Phalloceros sp. (34 indivíduos; $12 \%$ do total capturado - Espécie pouco abundante) (Figura 2). Por apresentarem abundância relativa menor que $10 \%$ do total capturado, as demais espécies foram classificadas como raras.

Astyanax intermedius é uma espécie característica de Mata Atlântica (CORREIA et al., 2007). Ocorre na bacia do rio Paraíba do Sul e rios costeiros do Estado do Rio de Janeiro, em trechos com águas claras, em especial no curso médio superior destes ambientes (BIZERRIL e PRIMO, 2001). Recentemente, Lezama et al. (2011) atribuíram a espécie à bacia do Rio Doce, Minas Gerais. Segundo Menezes et al. (2007), dados acerca de sua ecologia são escassos. Coswosck e Duboc (2011) estudando a dieta da espécie na bacia do rio Preto (ES) verificaram que sua dieta foi composta de itens de origem animal e vegetal, com insetos apresentando importância mais significativa, caracterizando a espécie como insetívora com hábito generalista. A espécie não é considerada ameaçada de extinção. 


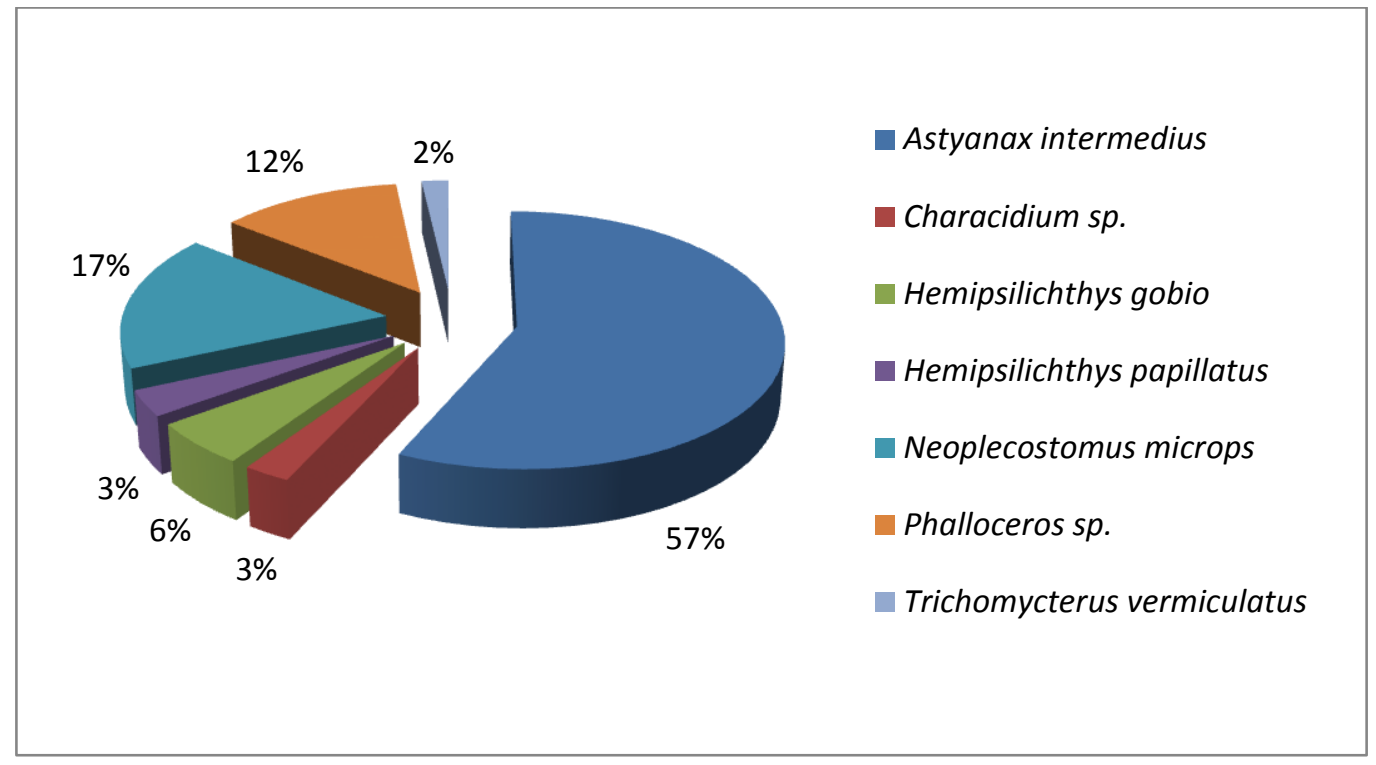

Figura 2: Abundância relativa das espécies de peixes registradas na área de influência da PCH Braço, durante o primeiro ano de operação do empreendimento.

Neoplecostomus microps ocorre no rio Paraíba do Sul e afluentes, além de pequenas bacias costeiras, entre os Estados do Rio de Janeiro e São Paulo (LANGEANI, 1990). É encontrada nas cabeceiras de rios e riachos em áreas de correnteza rápida e substrato arenoso ou rochoso (MENEZES et al., 2007). A espécie apresenta hábito bentônico e é desprovida de bexiga natatória (BRAGA, 2004), o que permite que vasculhe o substrato em busca de alimento. Braga et al. (2008) verificaram que a dieta de Neoplecostomus microps na microbacia do Ribeirão Grande, Serra da Mantiqueira Oriental (SP) é composta, principalmente, de larvas e ninfas aquáticas, prevalecendo as de Diptera e o período reprodutivo entre outubro e fevereiro, coincidindo com o período das chuvas. A espécie não é considerada ameaçada de extinção.

Hemipsilichthys gobio é conhecida de várias localidades da bacia do Rio Paraíba do Sul, nos Estados do Rio de Janeiro, Minas Gerais e São Paulo (REIS et al., 2006). Pode ser encontrada em rios de pequeno porte, com águas claras, de correnteza moderada a forte e substrato formado por rochas, pedras e cascalho. Pouco se conhece sobre sua ecologia. A espécie não é considerada ameaçada de extinção.

Registrada até então, apenas em duas localidades em um afluente do rio Preto, bacia do Rio Paraíba do Sul (MG) (REIS et al., 2006), Hemipsilichthys papillatus ocorre em rios de águas claras e correnteza moderada, com fundo formado por rochas, pedras e cascalho. Dados sobre sua ecologia também são escassos. A espécie não é considerada ameaçada de extinção.

Espécies do gênero Trichomycterus são amplamente distribuídas na região Neotropical (ROMÁN-VALENCIA, 2001). Apesar do grande número de espécies deste grupo, dados acerca da biologia das espécies (alimentação, reprodução ou dinâmica de populações) são escassos e muitas delas estão presentes em listas de espécies ameaçadas de extinção, como é o caso de Trichomycterus vermiculatus, considerado vulnerável no Estado do Rio de Janeiro (MAZZONI et al., 2000; BUCKUP et al., 2007). 
Duas das sete espécies registradas são classificadas em nível de gênero, o que reforça a necessidade de trabalhos taxonômicos sobre a ictiofauna Sul-Americana, conforme apontado por vários autores (e.g. BÖLKHE et al., 1978; MENEZES, 1992). Apesar das recentes descrições de espécies destes gêneros (Characidium, Phalloceros) (e.g. GRAÇA et al., 2008; LUCINDA, 2008), ainda há necessidade de estudos de cunho ecológico/naturalístico envolvendo ambientes fluviais de pequeno e médio porte. Segundo Menezes (1996) um dos aspectos que impedem a avaliação da distribuição da diversidade da ictiofauna de Mata Atlântica e demais regiões da América do Sul de maneira apropriada é a falta de conhecimento sobre composição taxonômica ao nível de espécie, de boa parte dos grupos representados. A ocorrência de espécies descritas recentemente e de outras ainda não descritas destaca o conhecimento limitado da ictiofauna de riachos do leste brasileiro (MIRANDA, 2009).

Apenas uma espécie (Trichomycterus vermiculatus) apresentou comprimento padrão superior a 15 centímetros (Tabela 2), de forma que as espécies capturadas na área de influência da PCH Braço podem ser caracterizadas como de pequeno porte (sensu CASTRO, 1999).

Tabela 2: Comprimento mínimo e máximo (em centímetros) dos indivíduos capturados na área de influência da PCH Braço, durante o primeiro ano de operação do empreendimento.

\begin{tabular}{cc}
\hline Espécies & $\begin{array}{c}\text { Comprimento } \\
\text { (min. - máx.) }\end{array}$ \\
\hline Astyanax intermedius & $3,4-11,2$ \\
Characidium sp. & $6,9-8,5$ \\
Hemipsilichthys gobio & $5,0-14,1$ \\
Hemipsilichthys papillatus & $6,8-9,0$ \\
Neoplecostomus microps & $4,2-9,3$ \\
Phalloceros sp. & $1,1-3,1$ \\
Trichomycterus vermiculatuls & $11,3-21,5$
\end{tabular}

Astyanax intermedius apresentou a maior biomassa dentre as espécies capturadas $(2089,4 \mathrm{~g}$.$) , seguida por Hemipsilichthys gobio (483,6 \mathrm{~g})$, Neoplecostomus microps $(482,1 \mathrm{~g}$.) e Trichomycterus vermiculatus (275,3g.). A biomassa total foi 3521,2g. (Tabela 3).

Tabela 3: Biomassa das espécies de peixes capturadas na área de influência da PCH Braço, durante o primeiro ano de operação do empreendimento.

\begin{tabular}{cccccc}
\hline Espécies & C1 & C2 & C3 & C4 & Total \\
\hline Astyanax intermedius & 350,5 & 76,8 & 617,2 & 1044,9 & $\mathbf{2 0 8 9 , 4}$ \\
Characidium sp. & 0,0 & 0,0 & 46,3 & 17,8 & $\mathbf{6 4 , 1}$ \\
Hemipsilichthys gobio & 38,4 & 122,8 & 319,4 & 3,0 & $\mathbf{4 8 3 , 6}$ \\
Hemipsilichthys papillatus & 14,1 & 35,1 & 42,9 & 28,8 & $\mathbf{1 2 0 , 9}$ \\
\hline
\end{tabular}




\begin{tabular}{cccccc}
\hline $\begin{array}{c}\text { Neoplecostomus microps } \\
\text { Phalloceros sp. }\end{array}$ & 62,0 & 47,5 & 285,3 & 87,3 & $\mathbf{4 8 2 , 1}$ \\
$\begin{array}{ccccc}\text { Trichomycterus vermiculatus } \\
\text { Total }\end{array}$ & 0,0 & 82,1 & 180,2 & 13,0 & $\mathbf{2 7 5 , 3}$ \\
\hline & $\mathbf{4 6 5 , 0}$ & $\mathbf{3 6 4 , 3}$ & $\mathbf{1 4 9 5 , 8}$ & $\mathbf{1 1 9 6 , \mathbf { 1 }}$ & $\mathbf{3 5 2 1 , 2}$ \\
\hline
\end{tabular}

Na comparação entre as campanhas de coleta, as campanhas 3 e 4 apresentaram maiores valores de riqueza e diversidade (Tabela 4). Os valores de riqueza e abundância das espécies capturadas são semelhantes aos registrados em outros riachos com características semelhantes ao rio do Braço: corpo d'água encaixado, com corredeiras e quedas, algumas muito acentuadas (e.g. MAZZONI et al., 2001). Tais características refletem a seletividade destes ambientes, nos quais são encontradas apenas espécies com adaptações que permitem a colonização e fixação nestes locais.

Tabela 4: Valores de diversidade, equitabilidade e riqueza registrados na área de influência da PCH Braço, durante primeiro ano de operação do empreendimento.

\begin{tabular}{ccccc}
\hline & C1 & C2 & C3 & C4 \\
\hline Diversidade & 0,761 & 1,461 & 1,562 & 0,971 \\
Equitabilidade & 0,535 & 0,862 & 0,681 & 0,377 \\
Riqueza & 4 & 5 & 7 & 7 \\
\hline
\end{tabular}

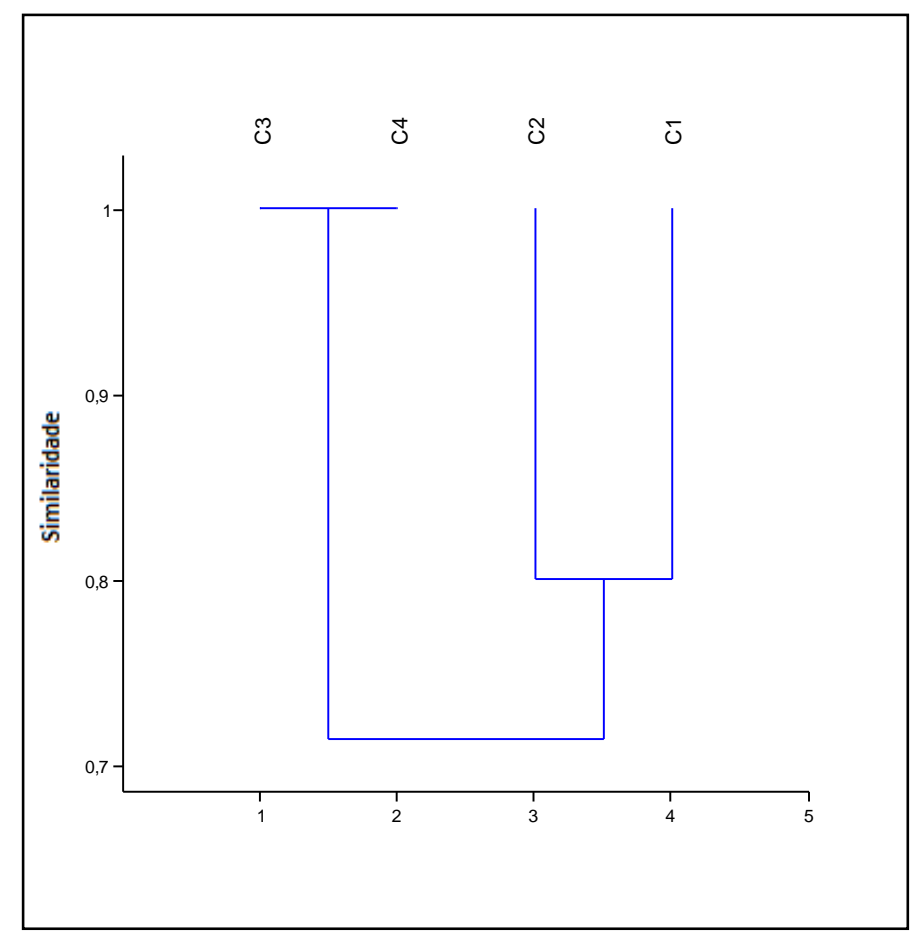

Figura 4: Análise de similaridade de Jaccard com base na presença/ausência das espécies nas campanhas de monitoramento no primeiro ano de operação da PCH Braço. 


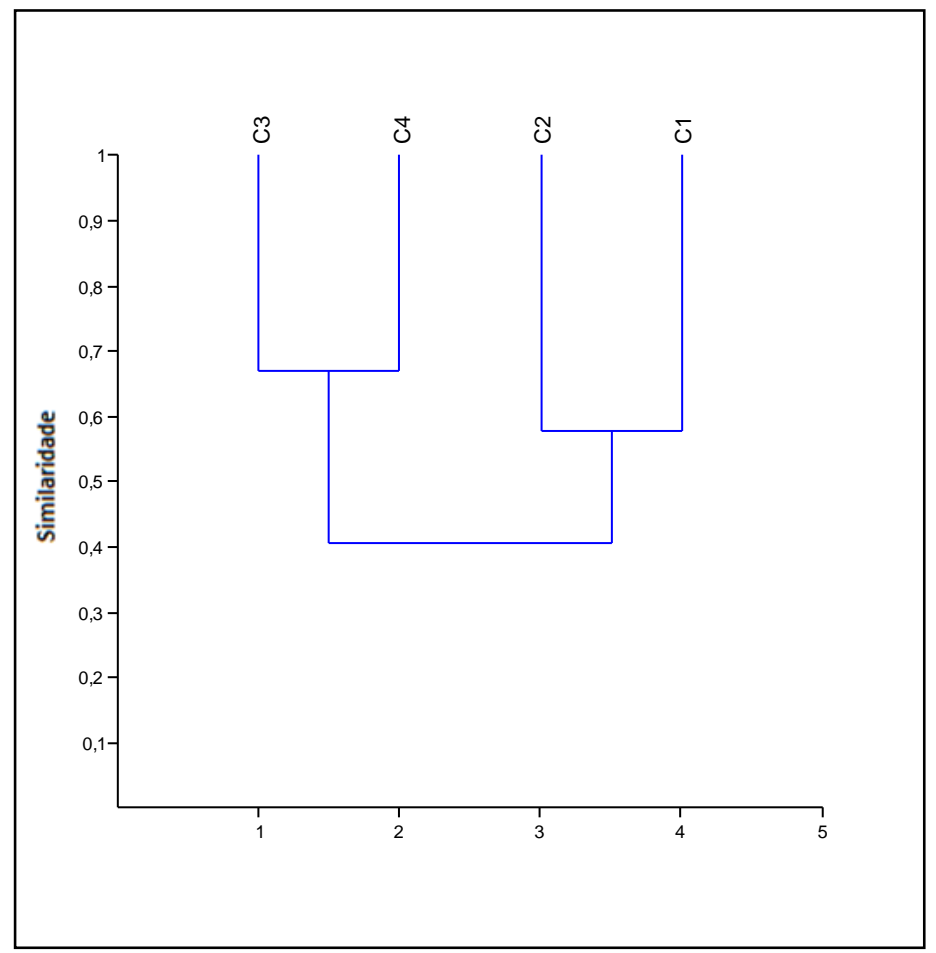

Figura 5: Análise de similaridade de Bray-Curtis com base nas abundâncias das espécies nas campanhas de monitoramento no primeiro ano de operação da PCH Braço.

As análises de agrupamento apresentam os níveis de coesão entre as campanhas amostrais com base nos dados de presença e ausência (Figura 4) e de abundância das espécies (Figura 5).

Os resultados das análises de similaridade indicam a separação das coletas em dois grupos. Quanto à similaridade de Jaccard, que leva me conta a presença e a ausência das espécies, as coletas realizadas em novembro de 2011 e fevereiro de 2012 apresentaram similaridade de 100\%, enquanto que as realizadas em maio e agosto de 2011 apresentaram similaridade de cerca de $80 \%$. No que se refere à similaridade de Bray-Curtis, os mesmos grupos foram formados, porém com valores menores.

\section{CONSIDERAÇÕES FINAIS}

A importância de estudos em áreas de influência direta de represamentos para a produção de energia elétrica tem sido destacada, em função do grande número desse tipo de empreendimento no Brasil e no mundo. Tais estudos devem ser conduzidos de forma a gerarem informações para o estabelecimento de ações de manejo com vistas à conservação destes ambientes e sua ictiofauna, uma vez que é esperado que o impacto decorrente de represamentos promova alterações na riqueza e abundância de espécies, em função da nova configuração do ambiente a partir da formação do reservatório e possível diminuição do fluxo da água em determinados trechos do rio. Até o momento, os dados obtidos no âmbito do programa de monitoramento de ictiofauna indicam que na área de influência direta da PCH Braço, tal condição não se aplica. Tal fato pode estar relacionado à localização do reservatório, a 840 metros de altitude e cerca de 30 metros a montante da foz do rio Jararaca (afluente da margem 
esquerda do rio do Braço), cujas descargas naturais garantem as vazões residuais (sanitárias) no trecho do rio do Braço entre a barragem e a casa de força (MOURA, 2012).

\section{AGRADECIMENTOS}

A Pequena Central Hidrelétrica Rio do Braço S/A e a Dossel Ambiental Consultoria e Projetos Ltda. pela disponibilização dos dados. A professora Dr.ㅁ Rosana Souza-Lima pelo auxílio na identificação do material.

\section{BIBLIOGRAFIA}

1. ARAÚJO, F. G. Adaptação do índice de integridade biótica usando a comunidade de peixes para o rio Paraíba do Sul. Revista Brasileira de Biologia, v. 58, n. 4, p. 547-558, 1998.

2. BIZERRIL, C \& PRIMO, P. B. Peixes de águas interiores do Estado do Rio de Janeiro FEMAR - SEMADS (Cooperação técnica Brasil-Alemanha, Projeto PLANÁGUA), Rio de Janeiro. 417p., 2001.

3. BÖHLKE, J.E., WEITZMAN, S. H. \& MENEZES, N. A. Estado atual da sistemática dos peixes de água doce da América do Sul. Acta Amazonica, v. 8, n. 4, p. 657-677, 1978.

4. BRAGA, F. M. S. Hábitat, distribuição e aspectos adaptativos de peixes da microbacia do Ribeirão Grande, Estado de São Paulo, Brasil. Acta Scientiarum, v. 26, n. 1 p. 31-36, 2004.

5. BRAGA, F.M.S. \& ANDRADE, P.M. Distribuição de peixes na microbacia do ribeirão Grande, Serra da Mantiqueira Oriental, São Paulo, Brasil. Iheringia, Série Zoologia, v. 95, n. 2, p. 121-126, 2005.

6. BRAGA, F.M.S., GOMIERO, L.M. \& SOUZA, U.P. Aspectos da reprodução e alimentação de Neoplecostomus microps, (Loricariidae, Neoplecostominae) na microbacia do Ribeirão Grande, serra da Mantiqueira oriental (SP). Acta Scientiarum, v. 30, n. 4, p. 455-463, 2008.

7. BRAY J.R. \& CURTIS J.T. An ordination of the upland forest communities of southern Wisconsin. Ecological Monographs, v. 27, p. 325-349, 1957.

8. BUCKUP, P.A.; MENEZES, N.A. \& GHAZZI, M.S. Catálogo das espécies de peixes de água doce do Brasil. Rio de Janeiro: Museu Nacional. 195 p., 2007.

9. CARVALHO, E.D. \& SILVA, V.F.B. Aspectos ecológicos da ictiofauna e da produção pesqueira do reservatório de Jurumirim (Alto do rio Paranapanema, São Paulo). p. 769800. In: Henry, R. (Ed.) Ecologia de Reservatórios: estrutura, função e aspectos sociais. FUNDIBIO; FAPESP, Botucatu, 1999.

10. CASTRO, R.M.C. Evolução da ictiofauna de riachos sul-americanos: padrões gerais e possíveis processos causais. p. 139-159. In: Caramaschi, E.P.; Mazzoni, R. \& P.R. PeresNeto (Eds). Ecologia de Peixes de Riachos. Série Oecologia Brasiliensis, Vol. VI. PPGEUFRJ. Rio de Janeiro, Brasil, 1999.

11. CORREIA, M. A. L., BRITO, M. F. G. \& CARAMASCHI, E. P. Biologia reprodutiva de Astyanax cf. intermedius Eigenmann, 1908 no rio Macaé, RJ. In: XI Congresso Brasileiro de Limnologia, Resumos, 2007. 
12. COSWOSCK, M.C. \& DUBOC, L.F. Ecologia alimentar de Astyanax intermedius (Characidae: Characiformes) na sub-bacia do rio Preto, bacia do rio São Mateus, ES. In: X Congresso de Ecologia do Brasil. Anais, 2011.

13. GRAÇA, W.J., PAVANELLI, C.S. \& BUCKUP, P.A. Two New Species of Characidium (Characiformes: Crenuchidae) from Paraguay and Xingu River Basins, State of Mato Grosso, Brazil. Copeia, v. 2008, n. 2, p. 326-332, 2008.

14. KUSMA, C.M. \& FERREIRA, F.W. Mecanismo de transposição de peixes de pequena central hidrelétrica. Ciência Rural, v. 40, n. 1, p. 89-94, 2012.

15. LANGEANI, F. Revisão do gênero Neoplecostomus Eigenmann \& Eigenmann, 1888, com a descrição de quatro novas espécies do Sudeste brasileiro (Ostariophysi, Siluriformes, Loricariidae). Comunicações do Museu de Ciências e Tecnologia PUCRS, Série Zoologia, v. 3, p. 3-31, 1990.

16. LEZAMA, A.Q., TRIQUES, M.L. \& QUEIROZ, F.M. Astyanax intermedius Eigenmann, 1908 (Actinopterygii: Characiformes: Characidae): Distribution extension in eastern Brazil. Check List, v.7, n. 5, p. 583-584, 2011.

17. LOWE-MCCONELL, R. H. Ecological Studies in Tropical Fish Communities. Cambridge, Cambridge University. 382p., 1987.

18. LUCINDA, P.H.F. Systematics and biogeography of the genus Phalloceros Eigenmann, 1907 (Cyprinodontiformes: Poeciliidae: Poeciliinae), with the description of twenty-one new species. Neotropical Ichthyology, v. 6, n. 2, p. 113-158, 2008.

19. MAGURRAN, A.E. Measuring biological diversity. Blackwell Publishing. 256 p., 2004.

20. MAZZONI, R., BIZERRIL, C.R.S.F., BUCKUP, P.A., CAETANO FILHO, O., FIGUEIREDO, C.A., MENEZES, N.A., NUNNAN, G.W. \& TANIZAKI-FERREIRA, K. Peixes. pp. 63-74. In: BERGALLO, H., ROCHA, C.F.D., SANTOS-ALVES, M.A. \& VAN SLUYS, M., A fauna ameaçada de extinção do Estado do Rio de Janeiro. Ed. UERJ, Rio de Janeiro. 168 p., 2000.

21. MAZZONI, R., TOSTA, G.C., PETITO, J., MIRANDA, J.C. \& SCHUBART, S.A. A Ictiofauna de água doce da Ilha Grande - RJ. In: XIV Encontro Brasileiro de Ictiologia, Resumos, 2001.

22. MELO, F.A.G. Revisão taxonômica das espécies do gênero Astyanax Baird \& Gaimard, 1854, (Teleostei: Characiformes: Characidae) da região da Serra dos Órgãos, Estado do Rio de Janeiro, Brasil. Arquivos do Museu Nacional, v. 59, p. 1-46, 2001.

23. MENEZES, N. A. Sistemática de peixes. In: Agostinho, A. A. \& Benedito-Cecilio, E. (Eds.) Situação atual e perspectivas da ictiologia no Brasil - Documentos do IX Encontro Brasileiro de Ictiologia. Maringá- Editora da UEM. 128p., 1992.

24. MENEZES, N.A. Padrões de distribuição da biodiversidade da Mata Atlântica do sul e sudeste brasileiro: peixes. In: Conservation International do Brasil, Fundação Biodiversitas, Fundação S.O.S. Mata Atlântica \& Fundação André Tosello - Wokshop sobre padrões de biodiversidade da Mata Atlântica do Sudeste e Sul do Brasil. Campinas, São Paulo. http://www.bdt.org.br,1996. (Consulta em 09/ix/2004).

25. MENEZES, N.A., WEITZMAN, S.H., OYAKAWA, O. T., LIMA, F.C.T. de, CASTRO, R.M.C., \& WEITZMAN, M.J. Peixes de água doce de Mata Atlântica: lista preliminar das espécies e comentários sobre a conservação de peixes de água doce neotropicais. Museu de Zoologia - Universidade de São Paulo. 408 p., 2007.

26. MIRANDA, J.C. Estrutura e padrões ecomorfológicos de comunidades de peixes de uma microbacia do Atlântico Leste, Rio de Janeiro. Tese (Doutorado). Universidade do Estado do Rio de Janeiro. 88 p., 2009. 
27. MIRANDA, J. C. Ameaças aos peixes de riachos da Mata Atlântica. Natureza On Line, v. 10, p. 136-139, 2012.

28. MOURA, T.D. Diagnóstico ambiental da Bacia hidrográfica do rio Jararaca, município de Bananal, São Paulo. Monografia. Universidade Federal Rural do Rio de Janeiro. 23 p., 2012.

29. MOYLE, P.B. \& MOUNT, J.F. Homogeneous rivers, homogeneous fauna. Proceedings off National Academy of Sciences, v. 104, n. 14, p. 5711-5712, 2007.

30. MYERS, N., MITTERMEIER, C.G., FONSECA, G.A.B. \& KENT, J. Biodiversity hotspots for conservation priorities. Nature, v. 408, p. 853-858, 2000.

31. PAVLOV, D.S. "Strutures assisting the migrations of non-salmonid fish": USSR. FAO Fisheries Technical Paper, v. 308, 97p. 1989.

32. PEREIRA, E.H.L., OLIVEIRA J.C. \& OYAKAWA O.T. Hemipsilichthys papillatus, a new species of loricariid catfish (Teleostei: Siluriformes) from Minas Gerais, Brazil. Ichthyological Exploration of Freshwaters, v. 11(4):377-383. 2000.

33. PEREZ-JÚNIOR, O.R. \& GARAVELLO, J.C. Ictiofauna do ribeirão do Pântano, afluente do rio Mogi-Guaçu, bacia do alto rio Paraná, São Paulo, Brasil. Iheringia, Série Zoologia, v. 97, n. 3, p. 328-335, 2007.

34. PETESSE, M.L. \& PETRERE JÚNIOR, M. As Barragens e os Peixes: O impacto das grandes hidrelétricas nas espécies dos rios represados. Ciência Hoje, v. 49, p. 30-35, 2012.

35. REIS, R.E., PEREIRA, E.H.L. \& ARMBRUSTER, J.W. Delturinae, a new loricariid catfish subfamily (Teleostei, Siluriformes), with revisions of Delturus and Hemipsilichthys. Zoological Journal of the Linnean Society, v. 147, p. 277-299, 2006.

36. ROMÁN-VALENCIA, C. Ecología trófica y reproductiva de Trichomycterus caliense y Astroblepus cyclopus (Pisces: Siluriformes) en el río Quindio, Alto Cauca, Colômbia. Revista de Biologia Tropical, v. 49, n. 2, p. 657-666, 2001.

37. SILVA NETO, A. P., GONÇALVES JUNIOR, L. P., NOGUEIRA, E. C., STOFFEL, S. M., JUNGER, E. N., MATIELO, M. D., PEREIRA, S. L. \& AMARAL, A. A. Riqueza ictiofaunística encontrada no Rio Norte, Bacia do Itapemirim. In: X Congresso de Ecologia do Brasil. Anais, 2011.

38. SHIBATTA, O. A., ORSI, M. L. \& BENNEMANN, S. T. Os peixes do Parque Estadual Mata do Godoy. p. 156-167. In: Torezan J. M. (Org.). Ecologia do Parque Estadual Mata do Godoy. Ed. Itedes, Londrina. 169 p., 2006.

39. TUNDISI, J. G. Reservatórios como sistemas complexos: teoria, aplicações e perspectivas para usos múltiplos. In: HENRY, R. ed. Ecologia de Reservatórios: estrutura, função e aspectos sociais. São Paulo, FUNDIBIO/ FAPESP. 800 p., 1999.

40. WWF. Dam right: Rivers at risk. WWF Dams Initiative. WWF International. Gland, Switzerland. 48 p., 2004. 